Cahiers $d u$ MONDE RUSSE

\section{Cahiers du monde russe}

Russie - Empire russe - Union soviétique et États indépendants

45/3-4 | 2004

Varia

\title{
Fedor Aleksandrovič Gajda, Liberal'naja oppozicija na putjah k vlasti (1914-vesna 1917 g.)
}

\section{Michel Tissier}

\section{OpenEdition}

\section{Journals}

Édition électronique

URL : https://journals.openedition.org/monderusse/4186

DOI : $10.4000 /$ monderusse.4186

ISSN : $1777-5388$

Éditeur

Éditions de l'EHESS

\section{Édition imprimée}

Date de publication : 1 juillet 2004

Pagination : 656-659

ISBN : 2-7132-2009-2

ISSN : $1252-6576$

Référence électronique

Michel Tissier, «Fedor Aleksandrovič Gajda, Liberal'naja oppozicija na putjah k vlasti (1914-vesna

1917 g.) ", Cahiers du monde russe [En ligne], 45/3-4 | 2004, mis en ligne le 02 juin 2009, consulté le 03 septembre 2022. URL : http://journals.openedition.org/monderusse/4186 ; DOI : https://doi.org/ 10.4000/monderusse.4186

Ce document a été généré automatiquement le 3 septembre 2022.

Tous droits réservés 


\title{
Fedor Aleksandrovič Gajda, Liberal 'naja oppozicija na putjah k vlasti (1914-vesna 1917 g.)
}

\author{
Michel Tissier
}

\section{RÉFÉRENCE}

Fedor Aleksandrovič GAJDA, Liberal'naja oppozicija na putjah k vlasti (1914-vesna 1917 g.) (L’opposition libérale en route vers le pouvoir, 1914-printemps 1917). Moscou, ROSSPEN, 2003, $432 \mathrm{p}$.

Le libéralisme politique et les libéraux suscitent de l'intérêt en Russie aujourd'hui, du moins chez les historiens. La publication de la thèse que le jeune historien russe Fedor Gajda a consacrée à l'action politique des libéraux russes dans les années 1914-1917 n'est pas un acte isolé. Elle constitue une manifestation supplémentaire de l'attention prêtée par les Russes, depuis le début des années 1990, à l'histoire de leurs premiers partis politiques, nés au début du xxe siècle et dont les luttes ont conditionné dans une large mesure le sort du pays en 1917. Les éditions moscovites Rosspen, qui publient le livre de F. Gajda, ont beaucoup contribué à faire connaître cet aspect longtemps négligé de l'histoire russe contemporaine, principalement à travers la publication de recueils de documents. L'ensemble éditorial que ces volumes composent a été jugé suffisamment remarquable pour qu'on parle à leur propos d'une «nouvelle histoire politique » ou, au moins, d'une renaissance de l'histoire politique en Russie ${ }^{1}$.

Le livre de F. Gajda est un produit individuel très abouti de cet intérêt collectif pour l'histoire politique, qui a fait une large place à l'étude des partis et des acteurs politiques de la tendance "libérale ", selon la terminologie de l'époque. Son travail a d'abord le mérite de se distinguer d'autres ouvrages récents consacrés aux "libéraux ", souvent limités à une approche biographique, si ce n'est hagiographique. Il manifeste en effet le 
souci de fournir une interprétation globale du rôle politique de ces libéraux dans la période de la guerre jusqu'au moment de leur arrivée au pouvoir.

$\mathrm{Au}$ point où commence son entreprise, l'auteur a affaire à deux traditions historiographiques principales. D'une part, il existe un schéma persistant, hérité des historiens soviétiques, mais qui a, ô combien, influencé leurs collègues étrangers, avec lequel F. Gajda rompt vigoureusement. Il consiste en l'idée d'une révolution de Février au caractère «bourgeois » par nature et révolutionnaire par erreur, qui suppose une sorte de progression nécessaire (et, bien entendu, triomphale) entre cet épisode et la seconde révolution, celle d'Octobre. L'un des buts de l'auteur est de réfuter ce schéma, en montrant d'abord que les " libéraux », en premier lieu les chefs du parti constitutionneldémocrate (les cadets), étaient tout, sauf des contre-révolutionnaires. Sa démonstration à cet égard est sans faille.

D'autre part, la recherche de F. Gajda peut également être située par rapport au débat récurrent, chez les historiens occidentaux cette fois, qu'avaient ouvert et nourri dès les lendemains de la révolution les plus notables des « libéraux » émigrés, au premier rang desquels V. Maklakov et P. Miljukov. Ce débat portait, au fond, sur la nature réelle du «libéralisme » russe des années 1905-1917, et en particulier sur l'idéologie du parti constitutionnel-démocrate. L'enjeu central en était la comparaison, explicite ou implicite, avec le modèle libéral occidental. F. Gajda n'est pas dupe des justifications $a$ posteriori apportées par les uns et par les autres à leurs actions personnelles, ni de leurs accusations réciproques. Mais son approche, qui caractérise le «libéralisme» russe de cette époque comme relevant fondamentalement d'un "radicalisme", sans rapport direct avec le libéralisme européen, s'inspire clairement des termes de cette controverselà. De plus, elle contredit surtout la version donnée par celui qui était le leader principal des cadets durant la période étudiée, P. Miljukov.

Toutefois, convaincu des limites de ces deux cadres historiographiques, et de leur ancrage dans une conjoncture idéologique qui appartient au passé, F. Gajda s'est fixé un objectif général d'un autre type. L'enjeu principal dans la compréhension de l'action politique des libéraux n'est pas, selon lui, de nature idéologique, ou plutôt il ne saurait être restreint à cet aspect-là. Il réside davantage dans la confrontation entre, d'une part, la conception du pouvoir que les «libéraux » ont développée, dans un contexte où le régime impérial apparaissait de plus en plus affaibli, et, d'autre part, leur comportement politique, aussi bien quand ils tentaient d'arriver au pouvoir qu'au moment où ils étaient effectivement en position de l'exercer. L'investigation de F. Gajda repose par conséquent sur la conviction que seule une approche pragmatique de l'histoire politique de la Russie en révolution est susceptible de fournir des éléments d'appréciation valables sur l'action politique des libéraux.

L'auteur propose ainsi une chronique de l'activité des personnalités et des groupes dirigeants libéraux, principalement ceux du parti constitutionnel-démocrate, dans l'opposition au régime tsariste, puis lors de leur passage au gouvernement. Il utilise pour ce faire des sources variées, avec une prédilection notable pour les comptes rendus des réunions politiques, jusque-là négligés au profit des déclarations officielles à usage externe. Il s'agit au final pour l'auteur d'expliquer quelle idée du " pouvoir » et de l'État s'est formée chez les cadets, et de décrire le «mécanisme du pouvoir» qui se met en place sous le gouvernement provisoire jusqu'au printemps 1917. F. Gajda se méfie de la confusion, trop fréquente selon lui chez les historiens, entre la dimension formelle, juridique en particulier, des déclarations convenues des libéraux sur «le 
gouvernement » et "la démocratie», et leur pratique effective du pouvoir. C'est la cohérence et l'efficacité de l'action politique qu'il veut jauger.

L'auteur dégage une première phase qui commence avec la guerre et qui débouche sur la naissance de la coalition parlementaire libérale dite du "bloc progressiste», en septembre 1915. Durant cette période, les cadets, et Miljukov au premier chef, ont montré selon lui leur volonté d'empêcher toute solution concrète de réorganisation d'un " pouvoir» dont ils ne voulaient plus. Pour eux, il n'était pas question de chercher un compromis avec le régime, mais il ne fallait surtout pas non plus que leurs concurrents libéraux, octobristes ou progressistes, y parvinssent de leur côté. C'est donc une logique essentiellement partisane qui est mise au jour, et F.Gajda le fait de manière très méticuleuse, en examinant surtout les rapports de force au sommet du parti et dans la concurrence avec les autres mouvements politiques, mais aussi en montrant les perceptions différentes entre militants politiques de province et dirigeants dans la capitale.

Pour les libéraux, une période d'incertitude succède aussitôt à la constitution du bloc progressiste, par suite du raidissement du régime qui n'est toutefois pas en état de le transformer en véritable "réaction ». F. Gajda repère surtout dans cette phase les éléments qui témoignent de l'attentisme des cadets, de leur adhésion à l'idée d'une révolution, toujours vue comme "inévitable " sans pour autant qu'ils s'y préparent le moins du monde. C'est toujours une forme de refus calculé des responsabilités qui caractérise leur stratégie, selon l'auteur. Bien plus, ce même trait se retrouve dans leur action « révolutionnaire » en février-mars 1917. Il en est ainsi de leur tentative, vouée à l'échec, de se prévaloir de la légitimité juridique de l'ancien ordre qu'ils avaient rejeté, quand ils tentent d'organiser la succession de Nicolas II et une forme de continuité du pouvoir. Les conséquences pratiques de cet état d'esprit chez les cadets se traduisent, selon l'auteur, dans la troisième phase qu'il étudie : celle de leur domination éphémère au sein du gouvernement. Là, ils œuvrent pour conserver à leur parti sa capacité d'influence, mais refusent d'assumer clairement les responsabilités du pouvoir. Ils ont tous les rouages en main, dit F. Gajda, mais ils n'en font rien et n'ont pas de plan. D'où leur impuissance devant la situation réelle du pays, et leur échec.

La démarche très pragmatique et chronologique que l'auteur a adoptée a une contrepartie. La ligne directrice de son exposé des faits repose parfois sur des présupposés qu'il n'explicite pas. C'est surtout le cas dans ce qui apparait comme le premier point de son raisonnement général, celui qui concerne la nature du « libéralisme » des cadets. F. Gajda juge l'action politique de ces derniers en soulignant ce qu'il voit comme une contradiction. Il y aurait, d'un côté, leur respect de principe, « doctrinal », pour la légalité et le "pouvoir légitime» et, de l'autre, leur inclination effective à privilégier la révolution pour arriver au pouvoir. De cette manière, l'auteur pense disqualifier le « libéralisme » des principaux dirigeants du parti constitutionneldémocrate. Ce faisant, il rejoint certains arguments développés dans la controverse née chez les émigrés russes après 1917 (et notamment par Victor Léontovitch dans son Histoire du libéralisme en Russie). Mais, là où l'on attendrait des précisions de l'auteur sur la signification à donner à la notion de "pouvoir légitime ", il fait seulement référence à une définition implicite du libéralisme, selon un modèle occidental qui apparaît plutôt schématique (idéalisé ?). Le courant libéral a sans doute été moins uniforme que ne le sous-entend l'auteur, aussi bien dans ses expressions doctrinales que dans ses traductions politiques en Europe au xixe et au xxe siècle, en particulier dans sa conception de la 
« légitimité » du pouvoir. L'action des libéraux russes gagnerait ainsi à être comparée au rôle des grands mouvements libéraux européens dans la lutte pour la "démocratisation » au xixe siècle.

Dans le second point capital de son raisonnement, qui présente les causes de l'échec très rapide des libéraux parvenus au pouvoir, l'auteur expose plus clairement ses critères de jugement. Et il emporte facilement l'adhésion. Toutes les incohérences et les faiblesses de la stratégie du parti constitutionnel-démocrate sont révélées. F. Gajda démontre de manière très neuve la prépondérance des intérêts partisans chez les dirigeants cadets et leur incapacité à prendre la mesure de leurs nouvelles responsabilités. La "Révolution ", selon lui, c'est donc surtout le moment de vérité pour les principaux libéraux russes, enfin mis en situation de montrer leurs talents, et que tout aurait dû préparer à assumer le pouvoir. Certains lecteurs pourront trouver à leur échec plus de circonstances atténuantes que ne le fait l'auteur, particulièrement sévère. Mais il n'est pas douteux que le cadre d'interprétation qu'il propose, fondé sur une documentation nombreuse étudiée avec un luxe de détails, soit le premier depuis longtemps à renouveler le débat sur les heurs et malheurs du libéralisme et de ses avatars en Russie².

\section{NOTES}

1. La revue américaine Kritika a proposé récemment un ensemble de recensions sur les différents volumes de documents publiés, dont une part importante est consacrée aux libéraux, et sur diverses monographies historiques récentes : "The new political history. Special issue ", Kritika. Explorations in Russian and Eurasian History, 5 (1), Winter 2004. Le livre de F. Gajda, publié en 2003 , n'est cependant pas pris en compte dans ce numéro.

2. Une traduction en anglais de quatre articles de F. Gajda, rédigés et publiés en russe durant les années de préparation de sa thèse, a été fournie dans le dossier intitulé « Russian liberals on the road to revolution and power, 1914-1917 » par la revue américaine Russian Studies in History, 41 (4), Spring 2003. 